\title{
Review of devices used in neuromuscular electrical stimulation for stroke rehabilitation
}

This article was published in the following Dove Press journal:

Medical Devices: Evidence and Research

24 August 2017

Number of times this article has been viewed

\author{
Kotaro Takeda' \\ Genichi Tanino² \\ Hiroyuki Miyasaka ${ }^{1,3}$ \\ 'Faculty of Rehabilitation, School \\ of Health Sciences, ${ }^{2}$ Joint Research \\ Support Promotion Facility, Center \\ for Research Promotion and Support, \\ Fujita Health University, Toyoake, \\ Aichi, ${ }^{3}$ Department of Rehabilitation, \\ Fujita Health University Nanakuri \\ Memorial Hospital, Tsu, Mie, Japan
}

\begin{abstract}
Neuromuscular electrical stimulation (NMES), specifically functional electrical stimulation (FES) that compensates for voluntary motion, and therapeutic electrical stimulation (TES) aimed at muscle strengthening and recovery from paralysis are widely used in stroke rehabilitation. The electrical stimulation of muscle contraction should be synchronized with intended motion to restore paralysis. Therefore, NMES devices, which monitor electromyogram (EMG) or electroencephalogram (EEG) changes with motor intention and use them as a trigger, have been developed. Devices that modify the current intensity of NMES, based on EMG or EEG, have also been proposed. Given the diversity in devices and stimulation methods of NMES, the aim of the current review was to introduce some commercial FES and TES devices and application methods, which depend on the condition of the patient with stroke, including the degree of paralysis.
\end{abstract}

Keywords: functional electrical stimulation, therapeutic electrical stimulation, EMG-triggered stimulation, brain-machine interface, brain-computer interface

\section{Introduction}

The clinical application of electrical stimulation is historical, with live torpedo fish being used to deliver electric current for pain treatment $\sim 2,000$ years ago. In more recent years, several implanted and non-implanted electrical stimulation devices have been widely used in a clinical setting. Examples of implanted devices include the following: artificial cardiac pacemakers, which are placed under the skin in the chest or belly to electrically stimulate cardiac muscle to control heart rhythms; ${ }^{1}$ cochlear implants placed in the inner ear, which electrically stimulate the auditory nerve corresponding to the frequency of the sound; ${ }^{2}$ deep brain stimulation, which delivers electrical impulses to specific brain areas to reduce tremor in Parkinson's or other movement disorders; ${ }^{3,4}$ spinal cord stimulators, which send a mild electric current to nerves in the spinal cord to mask a pain signal; 5 and non-implanted devices, including transcutaneous electrical nerve stimulation (TENS) and transcranial direct current stimulation (tDCS), which deliver electrical stimulation via electrode pads on the skin and scalp, respectively. For TENS, electrodes are often placed on the area of skin where the pain is present, and a low-voltage electrical current is delivered to treat a variety of painful conditions. ${ }^{6,7}$ tDCS is a noninvasive brain stimulation technique in which a weak direct current $(1-2 \mathrm{~mA})$ is applied from electrodes to the scalp, ${ }^{8}$ which excites or inhibits cortical excitability, ${ }^{9}$ depending on the polarity of electrode. In recent years, tDCS has been extensively studied in clinical neuropsychiatry and rehabilitation. ${ }^{10,11}$
Correspondence: Kotaro Takeda Faculty of Rehabilitation, School of Health Sciences, Fujita Health University, 98-I Dengakugakubo, Kutsukake-cho, Toyoake, Aichi 470-I 192, Japan

Tel +8I 562939000

Fax +8I 562936817

Email ktakeda@fujita-hu.ac.jp 
Artificially controlling human muscles or muscle nerves by neuromuscular electrical stimulation (NMES) is widely used in clinical rehabilitation for spinal cord injury and stroke, which often impair upper motor neurons and/or their neuronal pathways to lower motor neurons, consequently leading to paralysis of upper and/or lower limbs. Unilateral paralysis (ie, hemiparesis or hemiplegia) is particularly seen in many patients who survive a stroke. Recovery from the motor impairments may occur over weeks and months. The poststroke motor recovery is complex due to genetic, pathophysiologic, sociodemographic, and clinical factors. ${ }^{12}$ NMES is one of the therapeutic interventions that has been developed to try to induce the motor recovery. ${ }^{13}$ NMES can be used to stimulate the neuromuscular activity of the paretic limbs after stroke because normal electrical excitability often remains in lower motor neurons and their innervated muscles. Research on the use of NMES for rehabilitation has been increasing (Figure 1) since 1961, when Liberson et al ${ }^{14}$ stimulated the tibialis anterior to dorsiflex the ankle joint of patients with hemiplegia.

The purpose of NMES can be broadly classified into the following categories in general: functional electrical stimulation (FES), which, in a narrow sense, compensates for voluntary motion, and therapeutic electrical stimulation ${ }^{15}$ (TES), with the aim of muscle strengthening or recovery from paralysis. Although there are both implanted ${ }^{16-18}$ and non-implanted ${ }^{19}$ NMES devices, this review mainly focused on the non-implanted type that uses surface electrodes for stimulation and is often applied for stroke rehabilitation.

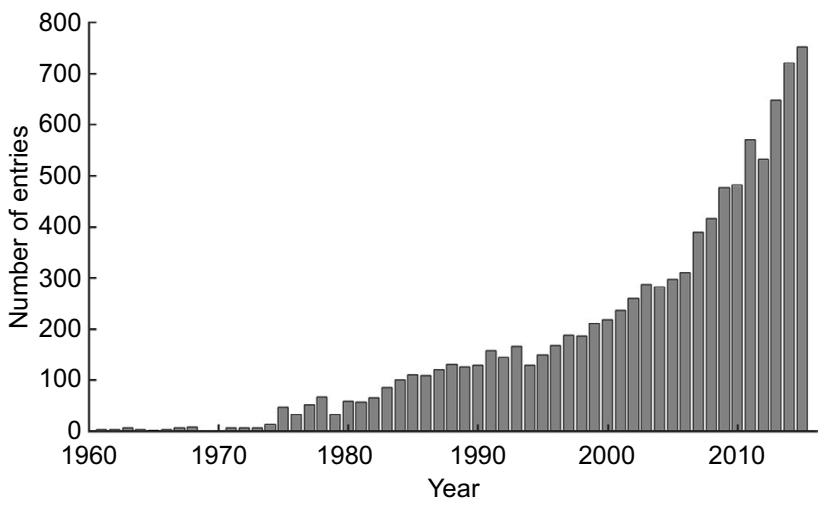

Figure I Results of a PubMed (service of the US National Library of Medicine [https://www.ncbi.nlm.nih.gov/pubmed/]) search for ("electrical stimulation" OR "electrical muscle stimulation" OR "electrical nerve stimulation" OR FES OR NMES) AND (stroke OR "cerebrovascular disease" OR hemiplegic OR hemiparetic OR hemiparesis OR paralysis OR rehabilitation).

Abbreviations: FES, functional electrical stimulation; NMES, neuromuscular electrical stimulation.

\section{Wave forms for NMES}

In NMES therapy, various stimulation parameters are used in the devices. Generally, as shown in Figure 2A, the waveform of the stimulation pulse may be monophasic, biphasic, and burst (polyphasic) waves. The pulse width is usually $150-300 \mu \mathrm{s}$, while the current intensity is dozens of milliampere. The pulse waveforms can be subdivided into rectangular waves, sine waves, etc. Biphasic waves, in contrast, can be further distinguished as symmetrical/asymmetrical or balanced/imbalanced (Figure 2B).

Muscle torque, fatigue, or pain induced by NMES depends on the wave parameters. Pain depends on the total amount of electrical charge delivered to the tissue. ${ }^{20}$ Petrofsky
Stimulus train

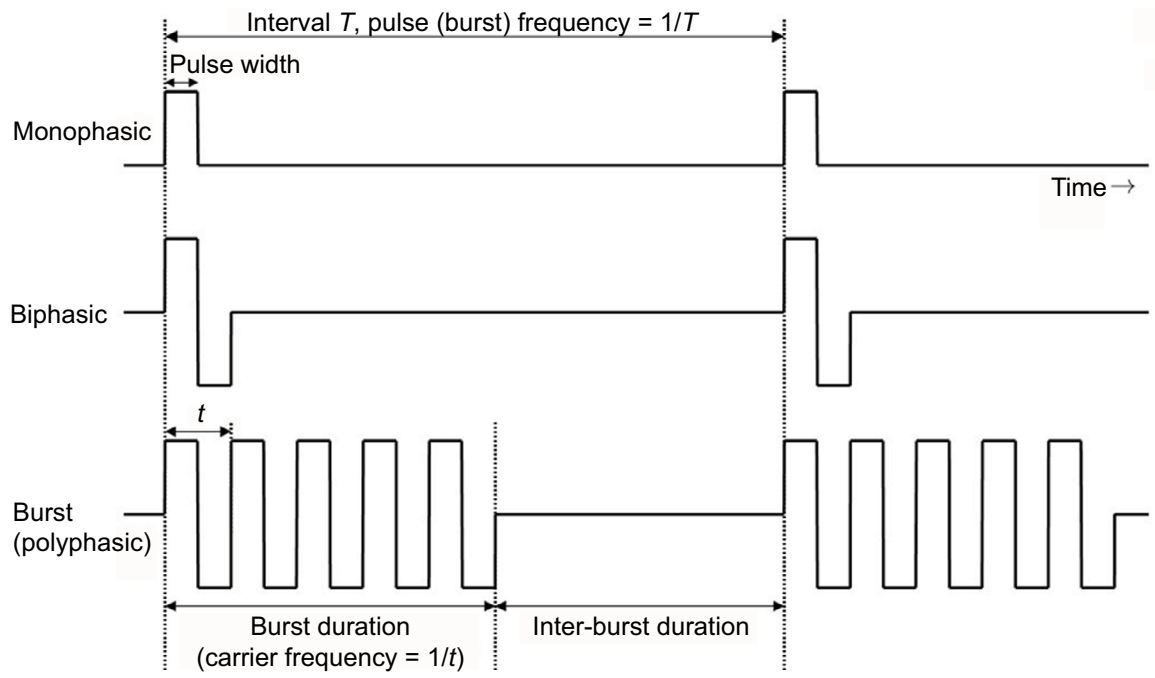

B

Wave forms

Half-cycle sine Exponentially climbing
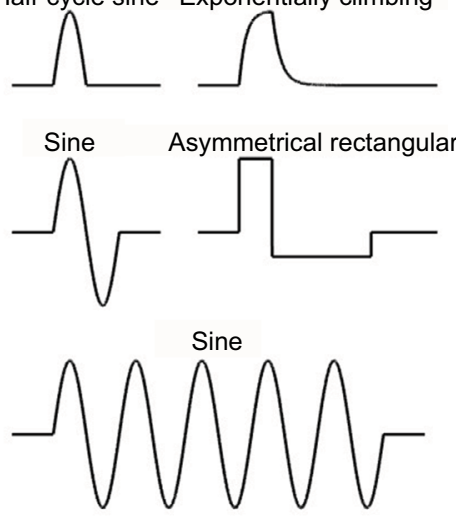

Figure 2 Parameters of NMES.

Notes: (A) Monophasic, biphasic, and polyphasic stimulus train. (B) Examples of wave forms. A specific stimulus, burst sine wave of carrier frequency of 2,500 $\mathrm{Hz}$ and burst and inter-burst duration of $10 \mathrm{~ms}$ is called a Russian current.

Abbreviation: NMES, neuromuscular electrical stimulation. 
et $\mathrm{al}^{21}$ showed that sine wave stimulation produced greater muscle strength, with less pain, in comparison with rectangular or polyphasic waves. Laufer et $\mathrm{al}^{22}$ also reported that monophasic and biphasic waveforms are more advantageous than burst waveforms and argued that the electrically induced fatigue is affected by the number of cycles per second, rather than the number of bursts per second. ${ }^{23}$ Therefore, monophasic or biphasic waveforms, rather than burst waveforms, may be more suitable for the clinical use of NMES. In fact, many NMES devices for stroke rehabilitation use the former.

\section{Functional electrical stimulation}

Although FES is a method that compensates for voluntary motion in a narrow sense, it cannot strictly be distinguished from TES. Many studies have demonstrated that rehabilitation training with FES improves activity of patients with stroke. ${ }^{24}$ The WalkAide system (Innovative Neurotronics, Inc., Austin, TX, USA) ${ }^{25}$ and NESS L300 (Bioness Inc., Valencia, CA, USA) ${ }^{26}$ are examples of typical commercial FES devices that are available for paralyzed lower limbs after stroke (Figures 3 and 4A, respectively). These devices are commonly used against forefoot dropping, which is a gait abnormality that occurs due to paralysis of the muscles of the lower leg (decreased ankle dorsiflexion). The drop foot during the swing phase (non-weight-bearing phase) of gait is one of the risk factors for a fall. ${ }^{27}$ In these devices, surface electrodes for stimulation are fixed by a cuff to the leg below the knee. Electrical stimulation is delivered during the

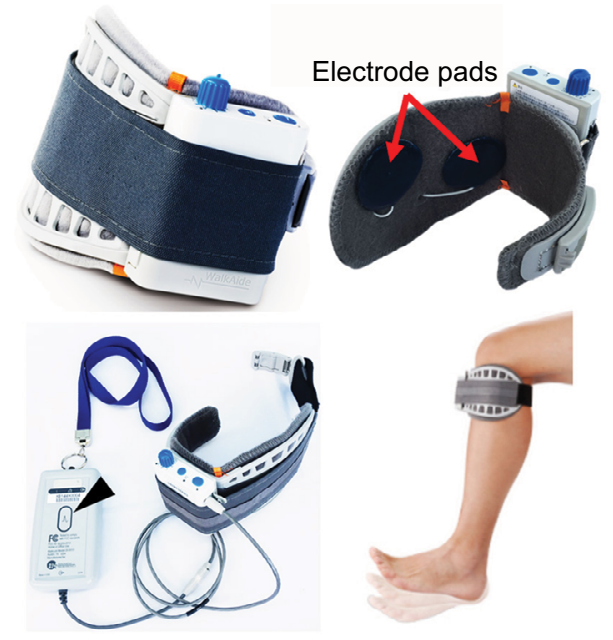

Figure 3 WalkAide system.

Notes: Surface electrode pads (red arrows) are fixed by a cuff to the leg below the knee. The stimulator is also tied to the cuff. Initially, a clinician connects a hand switch device (black arrow head) to the stimulator to dorsiflex the ankle manually during the swing phase of the patient's gait. The specific stimulation timing is then programmed, so that the electrical stimulation can be accurately performed consequently with the stimulator alone. A heel sensor under the insole of shoe or a tilt sensor built in the stimulator is used as a measure of the patient's gait. Image courtesy of Innovative Neurotronics, Inc., Austin, TX, USA.
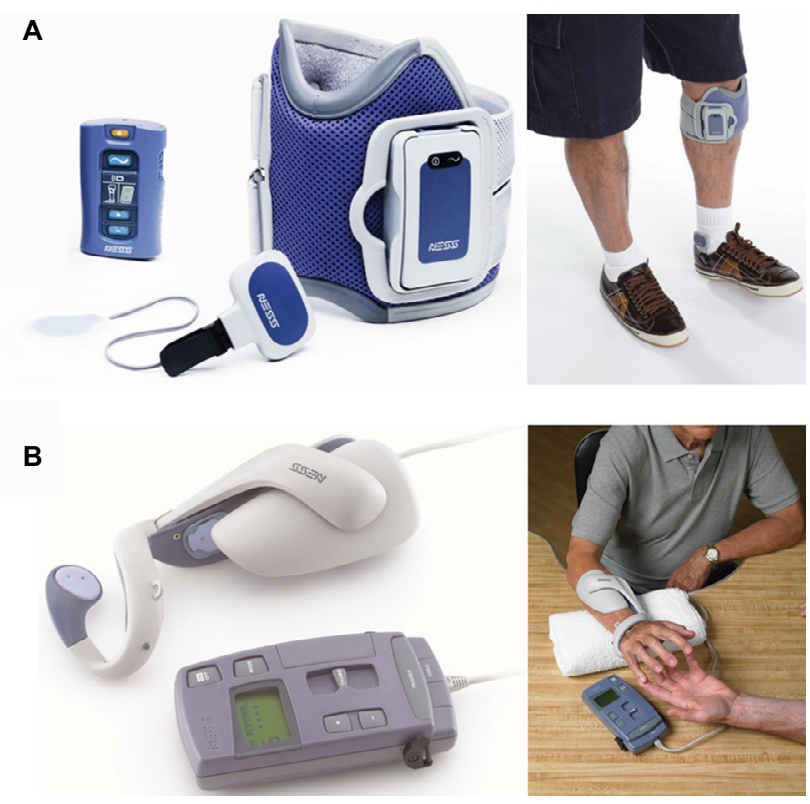

Figure 4 Commercial FES devices.

Notes: (A) NESS L300 and wireless foot switch for gait and (B) NESS H200, which is worn over the paralyzed arm and hand. Image courtesy of Bioness Inc., Valencia, CA, USA.

Abbreviation: FES, functional electrical stimulation.

swing phase of gait, producing ankle dorsiflexion to keep leg clearance. The heel contact and off (stance and swing phases) are detected by a tilt sensor placed with the knee cuff (WalkAide) or by a pressure sensor placed under the insole of shoe to determine stimulation periods (WalkAide and NESS L300).

Since NESS H200 (Bioness Inc.) ${ }^{28,29}$ is the only commercially available FES device for the upper limb and hand (Figure 4B), more robust and versatile devices are required for a wider group of people. ${ }^{30}$ Since hand or upper limb motion is more diverse in comparison to lower limb motion, the electrical stimulation system associated with stimulating the former is also complicated. The NESS H200 has five electrodes, which stimulate the five muscle groups of forearm and hand (ie, the extensor digitorum, extensor pollicis brevis, flexor digitorum superficialis, flexor pollicis longus, and thenar muscles) and implement both key gripping and palmar grasping.

\section{Therapeutic electrical stimulation}

Although some parameters of electrical stimulation (ie, stimulus position, stimulus intensity, pulse width, etc.) are adjusted according to the motion or condition of patient during FES, the stimulation methods for TES are even more varied.

Patients who have survived a stroke sometimes undergo electrical stimulation for muscle strengthening, although its use is not particular to stroke rehabilitation. In general, NMES 
often stimulates specific muscles, but in the case of muscle strengthening in stroke rehabilitation, multiple muscles, including those on the non-paralyzed side, are stimulated to enhance muscle strength. The reason for muscle strengthening in the non-paralyzed limb is to improve muscle weakness due to hospitalization (for example, knee extension force of the unaffected side decreases by $\sim 30 \%$ on the seventh day after the onset of stroke) $)^{31}$ or to acquire the compensatory strategies using the non-paralyzed limb. The belt electrode skeletal muscle electrical stimulation (B-SES) was also recently developed (Auto Tens Pro; Hormer Ion Co. Ltd., Tokyo, Japan) for muscle strengthening (Figure 5). For the B-SES, belt-like electrodes are wrapped around the waist, both knees, and both ankles to contract all lower limb skeletal muscles simultaneously. ${ }^{32}$ Since it is desirable to induce stronger muscle contraction to obtain sufficient muscle strengthening effect, the stimulus intensity is set to the limit that patients can endure. Therefore, B-SES adopts the exponentially climbing wave form shown in Figure 2B, which can produce greater muscle strength with less pain. ${ }^{33}$

In contrast, weak current NMES, which is approximately at sensory threshold or below motor threshold, has also been proposed as a possible supplemental therapy to facilitate motor function in patients with stroke. ${ }^{34}$ This is based on reports that somatosensory input enhances corticomotoneuronal excitability to the stimulated body parts. ${ }^{35,36}$ In this approach, rather than being used in isolation, NMES is applied in combination with rehabilitation training. The advantages of this approach are high safety, low pain, and high usability for various types of rehabilitation, including robot training (Figure 6). ${ }^{37}$

In the abovementioned TES approaches, the electrical stimulation is applied continuously and passively. However,

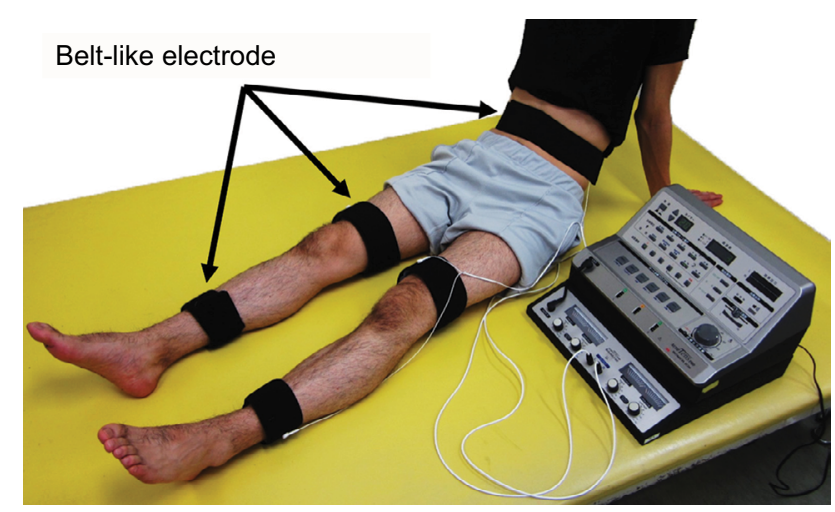

Figure 5 B-SES by Auto Tens Pro (Hormer lon Co. Ltd.).

Notes: Using the belt-like electrodes, B-SES can be used to stimulate all the muscles in the lower extremities, including the quadriceps femoris, hamstring, tibialis anterior, and triceps surae muscles. This device is currently used in Fujita Health University Nanakuri Memorial Hospital.

Abbreviation: B-SES, belt electrode skeletal muscle electrical stimulation.
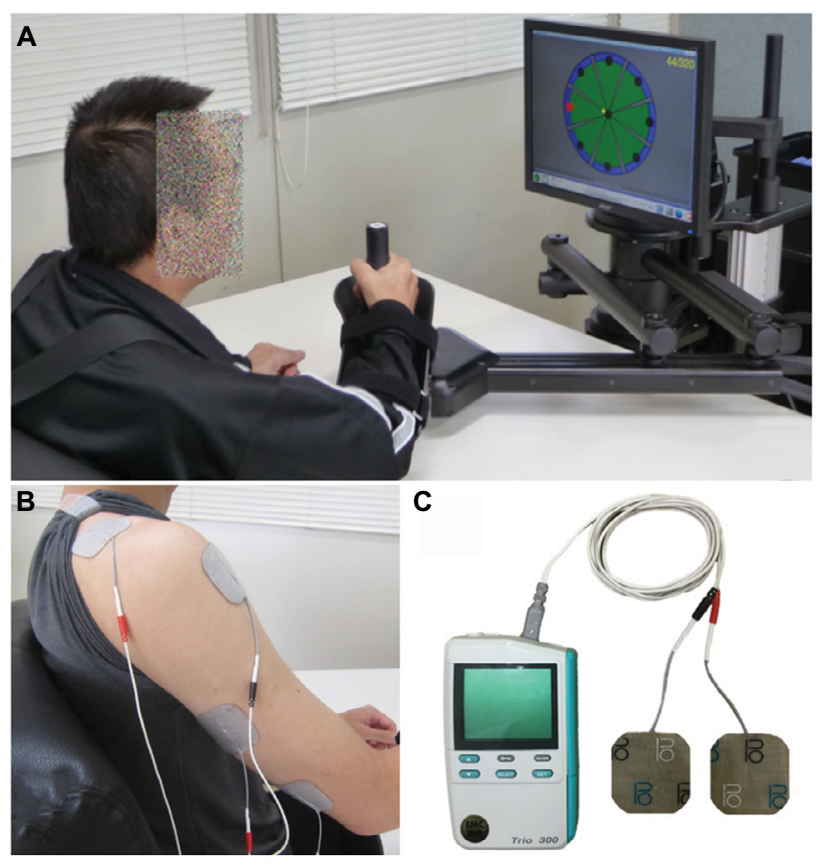

Figure 6 Robotic training for affected upper limb using MIT-Manus/InMotion2 system (Interactive Motion Technologies, Inc., Cambridge, MA, USA) (A) combined with NMES at sub-motor threshold intensity. ${ }^{37}$ NMES was delivered to the anterior deltoid and triceps muscles (B) using the Trio300 system (Ito Co. Ltd., Tokyo, Japan). (C) These devices are currently used in Fujita Health University Nanakuri Memorial Hospital.

Abbreviation: NMES, neuromuscular electrical stimulation.

for the motor recovery of paretic limbs after stroke, it is more common to perform NMES to encourage muscle contraction for an intended motion or to perform reciprocal NMES with agonists and antagonists. Rosewilliam et $\mathrm{al}^{38}$ recruited patients with stroke with no upper limb function and demonstrated that repetitive NMES for 30 minutes (on and off periods $=15 \mathrm{~s}$ ), which was applied twice in a working day for 6 weeks, to produce repetitive wrist extension, improved the wrist function of patients. Wu et $\mathrm{al}^{39}$ developed bilateral arm training combined with NMES for the triceps brachii muscle and anterior deltoid muscle in the affected arm. During the training, patients were required to move both their paralyzed and non-paralyzed arms simultaneously in the same way, and the NMES was triggered by the difference in movement of both arms to assist the motion. Osu et $\mathrm{al}^{40}$ used a surface electromyogram (EMG) of the unaffected hand as a clue for electrical stimulation of the paralyzed hand in a bilateral simultaneous motion. The EMG recorded by surface electrodes on the skin above skeletal muscle tissue is a common noninvasive method to assess the electrical activity that initiates muscle contraction and produces a physical force. .1,42 $^{4}$ For reciprocal NMES, alternate stimulation of dorsiflexors and plantarflexors according to the timing of gait improved the walking ability of patients with stroke. ${ }^{43,44}$ This therapeutic 
approach is similar to that of FES described earlier, which is triggered depending on the phase of gait. Essentially, there is little distinction between "TES" and "FES" in stroke rehabilitation.

Since adjusting the NMES to the timing of motion means synchronizing it to motor intention, EMG-triggered NMES has been developed. ${ }^{45-47}$ In this system, electrical stimulation is voluntarily triggered by EMG in the affected limb (the residual muscle activity of the paralyzed muscle). In recent years, EMG-triggered NMES has been combined with robot-aided rehabilitation, which has demonstrated improved motor function in patients with stroke. ${ }^{48,49}$ Furthermore, EMG-modulated NMES devices have also been developed, ${ }^{50-52}$ which controls not only the timing but also the intensity of electrical stimulation in direct proportion to the amount of residual voluntary EMG. Since Muraoka ${ }^{53}$ developed the device, where a pair of surface electrodes simultaneously records EMG from a muscle and stimulates the same muscle, EMG-modulated NMES can be applied even to a small muscle, in which it can be challenging to apply separate stimulating and recording electrodes. This NMES device, which is known as the integrated volitional control electrical stimulator (IVES), has been manufactured in Japan since 2008. ${ }^{54}$ The commercial IVES devices (PAS System and IVES+ System, OG Wellness Technologies Co., Ltd., Okayama, Japan) have two modes, ie, EMG-triggered NMES and EMG-modulated NMES (Figure 7).

In more recent years, there has also been an attempt to detect the motor intention using an electroencephalogram (EEG) instead of an EMG. Event-related desynchronization (ERD) and event-related synchronization (ERS), which respectively decrease and increase EEG frequency band power, are used to interpret the dynamics of brain oscillations $^{55}$ and are well known to be associated with motor attempt, motor imagery, or voluntary movement. ${ }^{56} \mathrm{~A}$ brain-computer interface (BCI) or brain-machine interface (BMI), a direct technological interface between the brain and a computer, based on the ERD and/or the ERS has recently been found to be a new tool to facilitate motor recovery after stroke. ${ }^{57}$ Clinical reports of stroke rehabilitation using a BCI system to trigger NMES (EEG-triggered NMES) for finger function, ${ }^{58}$ upper $^{59}$ and lower ${ }^{60}$ limb training, and gait rehabilitation ${ }^{61}$ have been published. Similar to the EMG-modulated NMES described earlier, an EEGmodulated NMES system has also been reported, which controls the current intensity of the NMES in a stepwise manner according to the appearance or disappearance of ERD. ${ }^{62}$ Although some of these BCI-NMES studies showed an improvement in paretic limbs of patients with stroke, currently, almost all are case reports or feasibility/safety studies. Thus, larger, controlled studies are warranted to validate the manufacture of commercial devices of EEGmodified NMES.

\section{Conclusion}

In stroke rehabilitation, NMES is used not only for muscle strengthening and motor recovery of paralyzed limbs as introduced in this review but also for reducing spasticity ${ }^{63}$ and improving swallowing function. ${ }^{64} \mathrm{With}$ the development of electronic engineering and clinical neuroscience, the devices and stimulation methods of NMES are diversifying. The application method of NMES differs depending on the condition of the patient with stroke, including the degree of paralysis. For patients with mild paralysis, weak NMES at sensory threshold or below motor threshold, combined with rehabilitation, may promote functional improvement. For moderate paralysis, EMG-triggered/modulated NMES may be a potential rehabilitative treatment option to restore
A

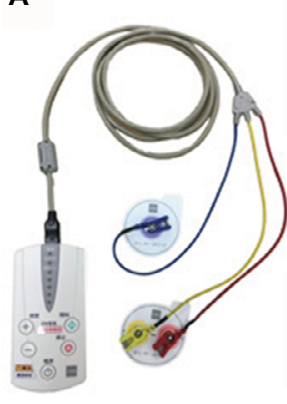

B

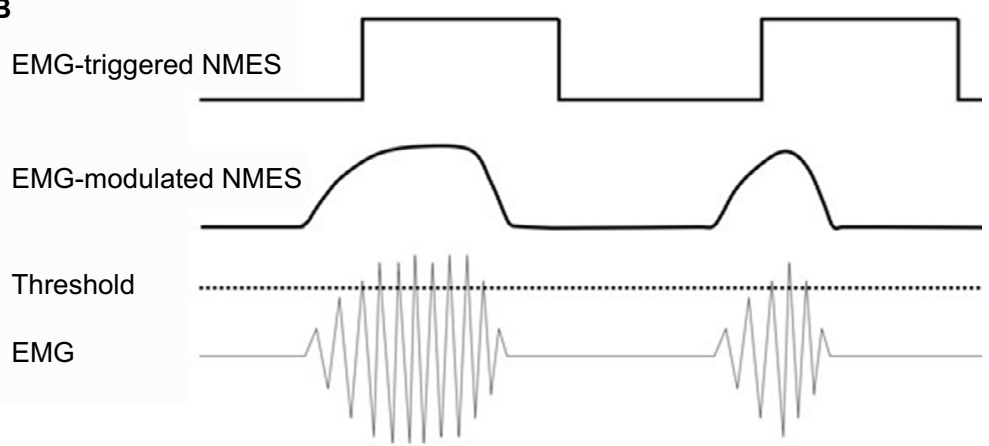

Figure 7 (A) The IVES+ system (OG Wellness Technologies Co., Ltd.) that is currently used in Fujita Health University Nanakuri Memorial Hospital. (B) EMG-triggered and EMG-modulated modes can be used for this device. In the former mode, NMES is applied with a constant current intensity for a fixed time when an EMG that exceeds a predefined threshold is detected. In the latter mode, the intensity of the stimulation current is proportional to the amplitude of EMG.

Abbreviations: IVES, integrated volitional control electrical stimulator; EMG, electromyogram; NMES, neuromuscular electrical stimulation. 
motor function and improve recovery. For severely affected patients in whom surface EMG is not detectable, EEGtriggered NMES may have therapeutic efficacy. Alternatively, in case of patients with severe paresis, in whom there is no motor intent, NMES aimed at muscle strengthening may be required to acquire compensatory movement in the non-paralyzed side.

Although the present review introduced some NMES devices and their clinical application, this was not a systematic review or a meta-analysis. The evidence of the applications of NMES in rehabilitation is still limited.$^{65}$ In particular, further research on recent new techniques, such as EEGtriggered/modulated NMES, using a controlled design is warranted. ${ }^{66}$ From the viewpoint of device development, there are many devices in which various stimulation parameters can be set with one device; however, there are few commercial devices that correspond to complicated operations such as hand movements. Further development and evidence-based commercial manufacture of EMG/EEG-triggered/modulated NMES devices are expected in the future.

\section{Disclosure}

The authors report no conflicts of interest in this work.

\section{References}

1. Nielsen JC, Gerdes JC, Varma N. Infected cardiac-implantable electronic devices: prevention, diagnosis, and treatment. Eur Heart J. 2015;36(37):2484-2490.

2. Roche JP, Hansen MR. On the horizon: cochlear implant technology. Otolaryngol Clin North Am. 2015;48(6):1097-1116.

3. Lozano AM, Gross RE. Introduction to deep brain stimulation. Neurosurg Focus. 2017;42(VideoSupp12):Intro.

4. Volkmann J. Deep brain stimulation for the treatment of Parkinson's disease. J Clin Neurophysiol. 2004;21(1):6-17.

5. Forrest DM. Spinal cord stimulator therapy. J Perianesth Nurs. 1996;11(5):349-352.

6. Claydon LS, Chesterton LS, Barlas P, Sim J. Dose-specific effects of transcutaneous electrical nerve stimulation (TENS) on experimental pain: a systematic review. Clin J Pain. 2011;27(7):635-647.

7. DeSantana JM, Walsh DM, Vance C, Rakel BA, Sluka KA. Effectiveness of transcutaneous electrical nerve stimulation for treatment of hyperalgesia and pain. Curr Rheumatol Rep. 2008;10(6):492-499.

8. Fregni F, Pascual-Leone A. Technology insight: noninvasive brain stimulation in neurology-perspectives on the therapeutic potential of rTMS and tDCS. Nat Clin Pract Neurol. 2007;3(7):383-393.

9. Jacobson L, Koslowsky M, Lavidor M. tDCS polarity effects in motor and cognitive domains: a meta-analytical review. Exp Brain Res. 2012;216(1):1-10.

10. Elsner B, Kugler J, Pohl M, Mehrholz J. Transcranial direct current stimulation (tDCS) for improving activities of daily living, and physical and cognitive functioning, in people after stroke. Cochrane Database Syst Rev. 2016;3:CD009645.

11. Brunoni AR, Nitsche MA, Bolognini N, et al. Clinical research with transcranial direct current stimulation (tDCS): challenges and future directions. Brain Stimul. 2012;5(3):175-195.

12. Alawieh A, Zhao J, Feng W. Factors affecting post-stroke motor recovery: implications on neurotherapy after brain injury. Behav Brain Res. In press 2016.
13. Langhorne P, Coupar F, Pollock A. Motor recovery after stroke: a systematic review. Lancet Neurol. 2009;8(8):741-754.

14. Liberson WT, Holmquest HJ, Scot D, Dow M. Functional electrotherapy: stimulation of the peroneal nerve synchronized with the swing phase of the gait of hemiplegic patients. Arch Phys Med Rehabil. 1961;42:101-105.

15. de Kroon JR, van der Lee JH, MJ IJ, Lankhorst GJ. Therapeutic electrical stimulation to improve motor control and functional abilities of the upper extremity after stroke: a systematic review. Clin Rehabil. 2002;16(4):350-360.

16. Shimada Y, Davis R, Matsunaga T, et al. Electrical stimulation using implantable radiofrequency microstimulators to relieve pain associated with shoulder subluxation in chronic hemiplegic stroke. Neuromodulation. 2006;9(3):234-238.

17. Marsolais EB, Kobetic R. Functional electrical stimulation for walking in paraplegia. J Bone Joint Surg Am. 1987;69(5):728-733.

18. Peckham PH, Keith MW, Freehafer AA. Restoration of functional control by electrical stimulation in the upper extremity of the quadriplegic patient. J Bone Joint Surg Am. 1988;70(1):144-148.

19. Broderick BJ, Breen PP, ÓLaighin G. Electronic stimulators for surface neural prosthesis. J Automat Contr. 2008;18(2):25-33.

20. Gracanin F, Trnkoczy A. Optimal stimulus parameters for minimum pain in the chronic stimulation of innervated muscle. Arch Phys Med Rehabil. 1975;56(6):243-249.

21. Petrofsky J, Laymon M, Prowse M, Gunda S, Batt J. The transfer of current through skin and muscle during electrical stimulation with sine, square, Russian and interferential waveforms. J Med Eng Technol. 2009;33(2):170-181.

22. Laufer Y, Ries JD, Leininger PM, Alon G. Quadriceps femoris muscle torques and fatigue generated by neuromuscular electrical stimulation with three different waveforms. Phys Ther. 2001;81(7):1307-1316.

23. LauferY, Elboim M. Effect of burst frequency and duration of kilohertzfrequency alternating currents and of low-frequency pulsed currents on strength of contraction, muscle fatigue, and perceived discomfort. Phys Ther. 2008;88(10):1167-1176.

24. Howlett OA, Lannin NA, Ada L, McKinstry C. Functional electrical stimulation improves activity after stroke: a systematic review with meta-analysis. Arch Phys Med Rehabil. 2015;96(5):934-943.

25. Everaert DG, Stein RB, Abrams GM, et al. Effect of a foot-drop stimulator and ankle-foot orthosis on walking performance after stroke: a multicenter randomized controlled trial. Neurorehabil Neural Repair. 2013;27(7):579-591.

26. van Swigchem R, Vloothuis J, den Boer J, Weerdesteyn V, Geurts AC. Is transcutaneous peroneal stimulation beneficial to patients with chronic stroke using an ankle-foot orthosis? A within-subjects study of patients' satisfaction, walking speed and physical activity level. $J$ Rehabil Med. 2010;42(2):117-121.

27. Weerdesteyn V, de Niet M, van Duijnhoven HJ, Geurts AC. Falls in individuals with stroke. J Rehabil Res Dev. 2008;45(8):1195-1213.

28. Alon G, Levitt AF, McCarthy PA. Functional electrical stimulation enhancement of upper extremity functional recovery during stroke rehabilitation: a pilot study. Neurorehabil Neural Repair. 2007;21(3): 207-215.

29. Alon G, Sunnerhagen KS, Geurts AC, Ohry A. A home-based, selfadministered stimulation program to improve selected hand functions of chronic stroke. NeuroRehabilitation. 2003;18(3):215-225.

30. Venugopalan L, Taylor PN, Cobb JE, Swain ID. Upper limb functional electrical stimulation devices and their man-machine interfaces. $J$ Med Eng Technol. 2015;39(8):471-479.

31. Harris ML, Polkey MI, Bath PM, Moxham J. Quadriceps muscle weakness following acute hemiplegic stroke. Clin Rehabil. 2001;15(3):274-281.

32. Numata H, Nakase J, Inaki A, et al. Effects of the belt electrode skeletal muscle electrical stimulation system on lower extremity skeletal muscle activity: evaluation using positron emission tomography. J Orthop Sci. 2016;21(1):53-56.

33. Tanino G, Tomita Y, Orand A, et al. Effect of rectangular and exponentially climbing waveforms on knee extension torque during neuromuscular electrical stimulation. Technol Disabil. 2016;28(4):139-144. 
34. Ikuno K, Matsuo A, Shomoto K. Sensory electrical stimulation for recovery of hand and arm function in stroke patients: a review of the literature. J Nov Physiother. 2012;S1:7.

35. Kaelin-Lang A, Luft AR, Sawaki L, Burstein AH, Sohn YH, Cohen LG. Modulation of human corticomotor excitability by somatosensory input. J Physiol. 2002;540(pt 2):623-633.

36. Hamdy S, Rothwell JC, Aziz Q, Singh KD, Thompson DG. Long-term reorganization of human motor cortex driven by short-term sensory stimulation. Nat Neurosci. 1998;1(1):64-68.

37. Miyasaka H, Orand A, Ohnishi H, Tanino G, Takeda K, Sonoda S. Ability of electrical stimulation therapy to improve the effectiveness of robotic training for paretic upper limbs in patients with stroke. Med Eng Phys. 2016;38(11):1172-1175.

38. Rosewilliam S, Malhotra S, Roffe C, Jones P, Pandyan AD. Can surface neuromuscular electrical stimulation of the wrist and hand combined with routine therapy facilitate recovery of arm function in patients with stroke? Arch Phys Med Rehabil. 2012;93(10):1715-1721.e1711.

39. Wu FC, Lin YT, Kuo TS, Luh JJ, Lai JS. Clinical effects of combined bilateral arm training with functional electrical stimulation in patients with stroke. IEEE Int Conf Rehabil Robot. 2011;2011:5975367.

40. Osu R, Otaka Y, Ushiba J, et al. A pilot study of contralateral homonymous muscle activity simulated electrical stimulation in chronic hemiplegia. Brain Inj. 2012;26(9):1105-1112.

41. Merletti R, Aventaggiato M, Botter A, Holobar A, Marateb H, Vieira TM. Advances in surface EMG: recent progress in detection and processing techniques. Crit Rev Biomed Eng. 2010;38(4):305-345.

42. Dieterich AV, Botter A, Vieira TM, et al. Spatial variation and inconsistency between estimates of onset of muscle activation from EMG and ultrasound. Sci Rep. 2017;7:42011.

43. Kesar TM, Perumal R, Reisman DS, et al. Functional electrical stimulation of ankle plantarflexor and dorsiflexor muscles: effects on poststroke gait. Stroke. 2009;40(12):3821-3827.

44. Embrey DG, Holtz SL, Alon G, Brandsma BA, McCoy SW. Functional electrical stimulation to dorsiflexors and plantar flexors during gait to improve walking in adults with chronic hemiplegia. Arch Phys Med Rehabil. 2010;91(5):687-696.

45. Cauraugh J, Light K, Kim S, Thigpen M, Behrman A. Chronic motor dysfunction after stroke: recovering wrist and finger extension by electromyography-triggered neuromuscular stimulation. Stroke 2000;31(6):1360-1364.

46. Kraft GH, Fitts SS, Hammond MC. Techniques to improve function of the arm and hand in chronic hemiplegia. Arch Phys Med Rehabil. 1992;73(3):220-227.

47. von Lewinski F, Hofer S, Kaus J, et al. Efficacy of EMG-triggered electrical arm stimulation in chronic hemiparetic stroke patients. Restor Neurol Neurosci. 2009;27(3):189-197.

48. Hu XL, Tong KY, Li R, et al. Post-stroke wrist rehabilitation assisted with an intention-driven functional electrical stimulation (FES)-robot system. IEEE Int Conf Rehabil Robot. 2011;2011:5975424.

49. Hu XL, Tong RK, Ho NS, Xue JJ, Rong W, Li LS. Wrist rehabilitation assisted by an electromyography-driven neuromuscular electrical stimulation robot after stroke. Neurorehabil Neural Repair. 2015;29(8):767-776.
50. Muraoka Y. Development of portable EMG-controlled electrical stimulator. In: Proc 41 st Soc Insturum Control Engnr Annu Conf; 2002; Osaka, Japan.; Vol. 3:2002-2007.

51. Muraoka Y, Tomita Y, Honda S, Tanaka N, Okajima Y. EMG-controlled hand opening system for hemiplegia. In: Proc 6th Vienna Int Workshop Funct Electrostimulation Basics Technol Appl; 1998; Vienna, Austria. 255-258.

52. Yeom H, Chang YH. Autogenic EMG-controlled functional electrical stimulation for ankle dorsiflexion control. J Neurosci Methods. 2010;193(1):118-125.

53. Muraoka Y. Development of an EMG recording device from stimulation electrodes for functional electrical stimulation. Front Med Biol Eng. 2002;11(4):323-333.

54. Muraoka Y, Tanabe S, Yamaguchi T, Takeda K. Specifications of an electromyogram-driven neuromuscular stimulator for upper limb functional recovery. Conf Proc IEEE Eng Med Biol Soc. 2013;2013:277-280.

55. Pfurtscheller G, Neuper C. Future prospects of ERD/ERS in the context of brain-computer interface (BCI) developments. Prog Brain Res. 2006;159:433-437.

56. Park W, Kwon GH, Kim YH, Lee JH, Kim L. EEG response varies with lesion location in patients with chronic stroke. J Neuroeng Rehabil. 2016;13:21.

57. Ushiba J, Soekadar SR. Brain-machine interfaces for rehabilitation of poststroke hemiplegia. Prog Brain Res. 2016;228:163-183.

58. Daly JJ, Cheng R, Rogers J, Litinas K, Hrovat K, Dohring M. Feasibility of a new application of noninvasive brain computer interface (BCI): a case study of training for recovery of volitional motor control after stroke. J Neurol Phys Ther. 2009;33(4):203-211.

59. Marquez-Chin C, Marquis A, Popovic MR. EEG-triggered functional electrical stimulation therapy for restoring upper limb function in chronic stroke with severe hemiplegia. Case Rep Neurol Med. 2016;2016:9146213.

60. Do AH, Wang PT, King CE, Abiri A, Nenadic Z. Brain-computer interface controlled functional electrical stimulation system for ankle movement. J Neuroeng Rehabil. 2011;8:49.

61. McCrimmon CM, King CE, Wang PT, Cramer SC, Nenadic Z, Do AH Brain-controlled functional electrical stimulation therapy for gait rehabilitation after stroke: a safety study. J Neuroeng Rehabil. 2015;12:57.

62. Takahashi M, Takeda K, Otaka Y, et al. Event related desynchronizationmodulated functional electrical stimulation system for stroke rehabilitation: a feasibility study. J Neuroeng Rehabil. 2012;9:56.

63. Sahin N, Ugurlu H, Albayrak I. The efficacy of electrical stimulation in reducing the post-stroke spasticity: a randomized controlled study. Disabil Rehabil. 2012;34(2):151-156.

64. Poorjavad M, Talebian Moghadam S, Nakhostin Ansari N, Daemi M. Surface electrical stimulation for treating swallowing disorders after stroke: a review of the stimulation intensity levels and the electrode placements. Stroke Res Treat. 2014;2014:918057.

65. Schuhfried O, Crevenna R, Fialka-MoserV, Paternostro-Sluga T. Non-invasive neuromuscular electrical stimulation in patients with central nervous system lesions: an educational review. J Rehabil Med. 2012;44(2):99-105.

66. Mukaino M, Ono T, Shindo K, et al. Efficacy of brain-computer interface-driven neuromuscular electrical stimulation for chronic paresis after stroke. J Rehabil Med. 2014;46(4):378-382.
Medical Devices: Evidence and Research

\section{Publish your work in this journal}

Medical Devices: Evidence and Research is an international, peerreviewed, open access journal that focuses on the evidence, technology, research, and expert opinion supporting the use and application of medical devices in the diagnosis, monitoring, treatment and management of clinical conditions and physiological processes. The identification of novel devices and optimal use of existing devices which will lead to improved clinical outcomes and more effective patient management and safety is a key feature. The manuscript management system is completely online and includes a quick and fair peer-review system. Visit http://www. dovepress.com/testimonials.php to read real quotes from authors. 